\title{
Plant-pollinator networks in semi-natural grasslands are resistant to the loss of pollinators during blooming of mass- flowering crops
}

Article

Accepted Version

Magrach, A., Holzschuh, A., Bartomeus, I., Riedinger, V., Roberts, S. P. M., Rundlöf, M., Vujić, A., Wickens, J. B., Wickens, V. J., Bommarco, R., González-Varo, J. P., Potts, S. G., Smith, H. G., Steffan-Dewenter, I. and Vilà, M. (2018) Plant-pollinator networks in semi-natural grasslands are resistant to the loss of pollinators during blooming of massflowering crops. Ecography, 41 (1). pp. 62-74. ISSN 0906-7590 doi: https://doi.org/10.1111/ecog.02847 Available at https://centaur.reading.ac.uk/71310/

It is advisable to refer to the publisher's version if you intend to cite from the work. See Guidance on citing.

Published version at: http://dx.doi.org/10.1111/ecog.02847

To link to this article DOI: http://dx.doi.org/10.1111/ecog.02847

Publisher: Wiley

All outputs in CentAUR are protected by Intellectual Property Rights law, including copyright law. Copyright and IPR is retained by the creators or other copyright holders. Terms and conditions for use of this material are defined in 
the End User Agreement.

www.reading.ac.uk/centaur

\section{CentAUR}

Central Archive at the University of Reading

Reading's research outputs online 


\section{Plant-pollinator networks in semi-natural grasslands are resistant to the loss of pollinators during blooming of mass- flowering crops}

5 Ainhoa Magrach ${ }^{1}$, Andrea Holzschuh ${ }^{2}$, Ignasi Bartomeus ${ }^{1}$, Verena Riedinger ${ }^{2}$, Stuart

6 P.M. Roberts ${ }^{3}$, Maj Rundlöf ${ }^{4}$, Ante Vujić ${ }^{5}$, Jennifer B. Wickens ${ }^{3}$, Victoria J. Wickens ${ }^{3}$,

7 Riccardo Bommarco $^{6}$, Juan P. González-Varo ${ }^{1,7}$, Simon G. Potts ${ }^{3}$, Henrik G. Smith ${ }^{4,8}$,

8 Ingolf Steffan-Dewenter ${ }^{2}$, Montserrat Vilà ${ }^{1}$

$9 \quad{ }^{1}$ Estación Biológica de Doñana (EBD-CSIC), Avda. Américo Vespucio s/n, Isla de la

Cartuja, 41092 Sevilla, Spain

${ }^{2}$ Department of Animal Ecology and Tropical Biology, Biocenter, University of

Würzburg, Am Hubland, 97074 Würzburg, Germany

${ }^{3}$ Centre for Agri-Environmental Research, School of Agriculture, Policy and

Development, University of Reading, Reading, RG6 6AR, UK

${ }^{4}$ Department of Biology, Lund University, 22362 Lund, Sweden

${ }^{5}$ Department of Biology and Ecology, Faculty of Sciences, University of Novi Sad, Trg

${ }^{6}$ Swedish University of Agricultural Sciences, Department of Ecology, 75007 Uppsala,

Sweden

${ }^{7}$ Conservation Science Group, Department of Zoology, University of Cambridge, The

David Attenborough Building, Pembroke Street, Cambridge, CB2 3QZ, UK

*Corresponding author: ainhoamagrach@ @otmail.com 
25 Keywords: Agroecosystems, plant-pollinator networks, floral resources, oilseed rape,

26 modularity, nestedness, null model, resilience, resource pulse

\section{Author contributions}

$28 \mathrm{AH}, \mathrm{ISD}, \mathrm{MR}, \mathrm{RB}, \mathrm{HGS}, \mathrm{SGP}$ and MV conceived and designed the study; AH

29 coordinated the study; MR, VR, JBW, VJW and JPGV collected field data; AM led data

30 analysis and drafted the manuscript; IB participated in data analyses and helped draft

31 the manuscript. All authors commented on manuscript drafts and gave final approval for

32 publication.

33

34 


\section{Abstract}

36 Mass-flowering crops lead to spatial redistributions of pollinators and to transient

37 shortages within nearby semi-natural grasslands, but the impacts on plant-pollinator interactions remain largely unexplored. Here, we characterised which pollinator species are attracted by oilseed rape and how this affected the structure of plant-pollinator networks in nearby grasslands. We surveyed 177 networks from three countries

41 (Germany, Sweden and United Kingdom) in 24 landscapes with high crop cover, and

42 compared them to 24 landscapes with low or no oilseed rape during and after crop

43 blooming. On average 55\% of grassland pollinator species were found on the crop,

44 which attracted 8-35\% of individuals away from grasslands. However, networks in the

45 grasslands were resistant to these reductions, since mainly abundant and highly mobile

46 species were attracted. Nonetheless, simulations indicated that network structural

47 changes could be triggered if $>50 \%$ of individuals were attracted to the crop (a value

48 well-above that found in our study system), which could affect community stability and resilience to further disturbance. 
Agricultural expansion and intensification are major drivers of land use change leading to species losses across natural and semi-natural ecosystems (Foley et al. 2005). These trends are set to continue given the constant growth in the world human population, currently projected to reach 9.1 billion by 2050 (FAO 2009). However, major expanses of agricultural land not only produce food, but also increasingly biofuel crops (Koh 2007). Within the EU, one of the fastest-growing biofuel crops for both energy production and food consumption is oilseed rape (Brassica napus L.) (FAO 2008), for which the area harvested has increased more than tenfold within Europe since the 1960s to $6,715,272$ ha in 2014 (FAO 2014).

Oilseed rape produces intense flushes of bright yellow insect-attractive flowers resulting in large spatio-temporal variation in the availability of floral resources at a landscape scale; around 525,000 plants/ha produce more than 100 flowers each during the peak flowering which lasts about 4 weeks (Hoyle et al. 2007). This large spike in oilseed flowering has implications for communities of native pollinators and the coflowering plants that rely on them (Westphal et al. 2003a, Holzschuh et al. 2013, 2016). Recent studies have suggested that although such a mass-flowering crop can enhance the abundance of pollinators at the landscape scale (Westphal et al. 2003b), the presence of this attractive resource can lead to a transient dilution of floral visitors in nearby habitats (Holzschuh et al. 2011, 2016). This dilution, caused by the attraction of pollinators from adjacent natural habitats into flowering crops, can alter the pollinator community composition (Diekötter et al. 2010) and reduce seed set in co-flowering wild plants (Holzschuh et al. 2011). But the effects on the network of interactions between the plants and their pollinators remain unexplored (Gonzalez-Varo et al. 2013), although this understanding is essential since the structure of the plant-pollinator network can 
affect community stability (Thébault and Fontaine 2010) and co-evolutionary dynamics (Guimarães et al. 2011).

Plant-pollinator networks are generally considered to be robust to disturbance (e.g., Nielsen and Totland 2014, Tiedeken and Stout 2015) given the redundancy in the number of pollinator species per plant species (Memmott et al. 2004), their nested structure (Bascompte et al. 2003, but see James et al. 2012), and the truncated powerlaw distribution followed by their number of links (Jordano et al. 2003), a consequence of morphological and phenological mismatching (Olesen et al. 2008, Bartomeus et al. 2016). However, as opposed to the way in which plant-pollinator networks disassemble in response to habitat loss (i.e. with specialist or rare species disappearing first, (Fortuna and Bascompte 2006, Aizen et al. 2012)), crop flowers do not attract all pollinators from the surrounding area equally. Rather, only a small number of common species carry out the bulk of crop pollination services (Kleijn et al. 2015). Thus, we hypothesised that networks in semi-natural habitats adjacent to mass-flowering crops will primarily lose common and generalist species which form the core of the network, and this could affect fundamental properties of the plant-pollinator networks. In particular, we expect the loss of generalist species from the network to decrease nestedness (i.e. specialist species tending to interact with a subset of those that interact with more generalist species) and evenness (i.e. leading to few strong interactions and many weak interactions) and it might increase complementary specialization (i.e. interaction exclusiveness). Such changes could be further reflected in an increase of network modularity due to the loss of many links across modules performed by these generalist pollinator species (Olesen et al. 2007). In a modular network, most pollinator species would interact preferentially with a subset of plant species within the community creating highly-connected units (or modules) with smaller probabilities of interacting 
with plant species within other units (Olesen et al. 2007). Taken together these shifts could result in less cohesive and more vulnerable networks (Bascompte et al. 2003).

We use a unique dataset from three European countries (Germany, Sweden and UK) to examine how the proportion of an insect-dependent mass-flowering crop (oilseed rape) in the landscape affects plant-pollinator networks in adjacent semi-natural grasslands at two time periods: during and after crop flowering. Our study addressed the following questions: (i) which species are attracted by oilseed rape flowers during peak flowering and what proportion of the whole pollinator community do they represent? (ii) what is the effect of such pollinator attraction on network structure in the seminatural grasslands? (iii) is there a particular level of pollinator loss that affects network structure and, if so (iv) how does this level compare to the current levels of pollinator reductions suffered at our study sites? We predicted that the greatest differences in pollinator community composition and plant-pollinator networks would occur in landscapes with high oilseed rape crop cover, during crop flowering, when generalist pollinators would first move away from the grasslands, to then return after massflowering ceases.

\section{Material and methods}

\section{Experimental design and data collection}

In each of three countries, Germany, Sweden and the United Kingdom, (Fig. 1a), we selected 16 semi-natural grassland sites with at least one autumn sown oilseed rape (OSR) field within $1 \mathrm{~km}$ (except in two cases where the nearest OSR field was located < $4 \mathrm{~km}$ away). Eight sites were located in landscapes with high relative cover for the region of OSR $(>6 \%,>11 \%$ and $>9.4 \%$ in the case of Germany, Sweden and UK respectively) while the remaining eight were located in landscapes of low cover of OSR 
125 (or no cover in the two sites as mentioned above, Table S1). Within a country, sites were selected to have similar geographical and land-use characteristics with differences

127 in OSR cover. At each study site we mapped the landscape within a $1 \mathrm{~km}$ radius surrounding each site. The radius was selected to cover the majority of forage flight distances and landscape-scale species responses (Steffan-Dewenter and Kuhn 2003, Holzschuh et al. 2011, Hanke et al. 2014). We calculated the proportion of the surface occupied by OSR and semi-natural habitats including extensively managed grasslands, calcareous grasslands, shrublands or forested areas. Semi-natural habitats were selected based on expert judgement to provide nesting sites, floral resources or refuges for pollinators. Across all sites, the proportion of the landscape covered by the OSR ranged from $0 \%$ to $42 \%$ and for semi-natural habitat from $2 \%$ to $32 \%$ (Table S1). There was a low covariation between the two land-uses $\left(\mathrm{R}^{2}<0.5\right.$ in all countries $)$. 
calculated flower cover for each grassland as the sum of flower units multiplied by the size of these flower units and divided by transect area for every species in the transect surveyed.

The autumn-sown OSR field site located within 1-km from each grassland site was surveyed for floral visitors twice during OSR flowering within the two transects as described previously but set parallel to the edge and at the interior ( $>25$ meters from the edge) of the crop. OSR fields and semi-natural grasslands were surveyed on the same day for data comparability. All transect surveys were conducted in temperatures above $17^{\circ} \mathrm{C}$, with no rain and low wind.

\section{Pollinator community}

We first evaluated sampling completeness of both the pollinator community and the plant-pollinator links using the Chao1 estimator of asymptotic species richness for abundance data (Chao 1984), a non-parametric estimator based on the frequency of rare species (or links) in the original sampling data. For each country, we first estimated the richness of pollinator species and plant-pollinator links accumulated as sampling effort increased up to $100 \%$ sampling coverage using package iNEXT (Hsieh et al. 2016). Secondly, we calculated the proportion of pollinator species and links recorded in our survey as compared to one with full sampling coverage. Thirdly, we evaluated which species were shared between grasslands and the crop as well as the proportion of pollinator species and individuals they represented within the grasslands out of the total pollinators. In order to assess which pollinator species were attracted to the crop during flowering we compared pollinator species sampled at the crop with those found in the adjacent grassland at that time period. We expected pollinator species attracted to the crop during flowering to decrease in abundance within grasslands surrounded by high 
174 OSR covers and to return to the grasslands after crop flowering while showing no 175 changes within landscapes with low OSR covers (Fig. 1c). Thus, we expect differences 176 in the abundance of each pollinator species between both types of grasslands only

177 during OSR flowering, when pollinators from grasslands surrounded by high OSR

178 covers will be attracted to the crop. We therefore assessed which species are attracted to 179 the crop by calculating their likelihood of being attracted as: $A t_{i}=1-\frac{H \cdot d u r_{i}}{L_{\cdot d u r_{i}}} \quad$ [Eqn.

180 1], where H.dur $r_{i}$ and $L . d u r_{i}$ represent pooled pollinator abundances within semi-

181 natural grasslands surrounded by high $(H)$ and low $(L)$ OSR proportions respectively for 182 each country during crop flowering for species $i$. This index equals 0 when $H . d u r_{i}=$ $183 L . d u r_{i}$ (no attraction), takes positive values up to 1 when, as hypothesized, $H . d u r_{i}<$ $184 L . d u r_{i}$ and negative values when $H . d u r_{i}>L . d u r_{i}$, which occurs for pollinator species 185 that are not attracted by the crop. In addition, for each country we evaluated the extent 186 of total pollinator attraction (TAt), i.e., the total share of the pollinator community within grasslands surrounded by high OSR cover that is attracted towards the crop during flowering. We did this by computing the proportion of all shared pollinator species $(n)$ found in grasslands surrounded by low OSR cover during crop flowering (L.dur, which we consider a spatial and temporal control) that were still present in grasslands surrounded by high OSR cover during the same period, when pollinators were being attracted to the crop (H.dur), TAt $=1-\frac{\sum_{i=0}^{n} H . d u r}{\sum_{i=0}^{n} L . d u r}$ [Eqn. 2].

\section{Plant-pollinator networks}

To analyse how the observed changes in the pollinator community affected network structure, we constructed a weighted interaction network for each 'grasslandperiod-year' by pooling data across transects and surveys. We built quantitative networks to represent the frequency of pollinator visits to plants (Fig. 1c), generating 
192 networks (i.e. 3 countries $x$ [ 8 high OSR +8 low OSR landscapes] $\times 2$ periods $\times 2$ years). Link density for a subset of networks (15) was too low (e.g., only one interaction observed due to very low flower cover) so these were omitted from the analysis.

We calculated the following network-level metrics: link density, interaction evenness, network-level complementary specialization $\left(\mathrm{H}^{\prime}{ }_{2}\right)$, modularity, and nestedness. We selected these metrics because although they are weakly correlated (Table S2) they reveal the diversity (i.e. link density and interaction evenness) and the relative distribution of interactions (i.e. complementary specialization, nestedness, and modularity) allowing for a broad understanding of flowering pulse effects on plantpollinator networks (Kaiser-Bunbury and Blüthgen 2015). These metrics are considered reliable indicators of network stability and robustness to species losses (Bascompte et al. 2003, Fortuna and Bascompte 2006, Bascompte and Jordano 2007, Olesen et al. 2007, Bastolla et al. 2009), although the role of some of them in stability is still under debate (e.g., nestedness, James et al. 2012). The weighted versions of these metrics were used due to the effect of matrix size, species abundances and each species' quantitative importance (a function of the frequency with which it interacts with other species in the network, (Kaiser-Bunbury and Blüthgen 2015)) on many of the network metrics (Blüthgen et al. 2007). We estimated link density as the weighted number of interactions per species, calculated as the marginal diversity of interactions per species weighted by the total diversity (Bersier et al. 2002). Interaction evenness was calculated following Tylianakis et al. (2007), where a higher number indicates a more even distribution of species interactions. Complementary specialization $\left(\mathrm{H}_{2}^{\prime}\right)$ measures the deviation of interaction frequencies from a completely generalized network $\left(\mathrm{H}_{2}^{\prime}=0\right)$ to a completely specialized one $\left(\mathrm{H}_{2}^{\prime}=1\right)$ (Blüthgen et al. 2007). Further, we calculated modularity using the QuanBiMo algorithm (Dormann and Strauss 2014), where the 
value represents the probability of showing more within-module than between-module interactions. This algorithm used to calculate modularity follows a stochastic approach and hence can lead to different modularity values in different runs. We thus ran the algorithm ten times and found an average difference between the first run and all subsequent runs of 0.02 only for a subset of the networks considered $(\mathrm{N}=15)$, while the value was consistent for the rest. Therefore, given low differences we report the results from a single run. Finally, we estimated nestedness using the weighted NODF (Nestedness based on Overlap and Decreasing Fill) metric (Almeida-Neto and Ulrich 2011), where a larger value indicates specialists have a higher tendency to interact with a perfect subset of the species that generalist species interact with.

The weighted version of these metrics can be affected by network size and the number of links, particularly in the case of complementary specialization, modularity or nestedness (Schleuning et al. 2012, 2014, Dormann and Strauss 2014). This can be problematic in comparisons of networks obtained with different sampling efforts or methodologies. In our study the weighted version of metrics is, however, unlikely to be affected due to the standardised sampling protocol and effort across all countries and hence raw values could be used. However, we additionally calculated and present corrected metrics for comparison with our raw metrics by standardising the raw values

$241\left(m_{\text {corr }}=\frac{\left.m_{\text {observed }}-\overline{m_{\text {null }}}\right)}{\sigma m_{\text {null }}}\right)$ using values obtained from 1000 null model algorithms (as recommended by (Dormann and Strauss 2014) and using the Patefield and vaznull network to evaluate whether the changes we observe in our raw metrics are primarily driven by changes in the number of species or in network connectance. 

evaluate whether species changed their role within the networks during OSR flowering. Species-level metrics were: normalised degree, species-level specialization ( $\left.d^{\prime}\right)$, withinmodule degree (z) and between-module connectivity (c), and nested rank. Normalised degree represents the actual number of plant partners a pollinator has compared to the total pool of potential plant partners. Species-level specialization represents a standardized form of the Kullback-Leiber distance (Blüthgen et al. 2006) which considers interaction frequencies whilst accounting for the diversity of partners and their availability. Higher values indicate greater levels of specialization or partner exclusiveness. Within-module degree ( $\mathrm{z}$ ) and between-module connectivity (c) were computed using the QuanBiMo algorithm previously used to calculate modularity. Both metrics were calculated as the number of links (within modules for $\mathrm{z}$ and between modules for c, Dormann and Strauss 2014). Nested rank rearranges a network by its maximal nestedness and quantifies the generalism of a given species through its rank in the matrix with increasing values for more specialist or rare species (Alarcón et al. 2008). These network metrics at the species level (except for $\mathrm{z}$ and c) were calculated using the specieslevel function in the bipartite package (Dormann et al. 2009).

\section{Data analyses}

We first evaluated whether the composition of the pollinator community changed with land use type and period by creating an ordination of sites based on the

267 similarity in the pollinator community composition recorded per site using the Bray-

268 Curtis index (Magurran 2004) followed by a non-metric multidimensional scaling (NMDS, Clarke and Warwick 2001). We then assessed actual differences by means of a permutational multivariate analysis of variance with distance matrices between sites. 
272 (i.e. link density, interaction evenness, complementary specialization, modularity and

273

274 nestedness) we used general linear mixed models (GLMMs) fitted for each country separately. Plant-pollinator networks were mapped per site, period and year based on pooled data from the respective two transects at each of the two surveys per site, period and year. Fixed effects were the proportion of OSR and semi-natural habitats in the landscape, flower cover, year, and period (during vs. after) as well as the two-way interactions of period with OSR, semi-natural habitat proportion and flower cover, and that of year with OSR, semi-natural habitat proportion and flower cover. Site was included as a random factor to account for non-independence of the repeated sampling in surveys carried out across two periods and years. All continuous variables were scaled prior to fitting models.

We ran all combinations of models using the dredge function in the MuMIn package (Barton 2013) and selected the best model based on the lowest second-order Akaike information criterion values (AICc). If more than one plausible model existed (i.e. when $\triangle \mathrm{AICc}<6$ for more than one model, Burnham et al. 2011) we computed average estimates for each variable across all models in which each variable was retained. We did not use shrinkage when estimating the average estimates for each variable, so that values were calculated only across models where the variable was retained. This modelling approach was used across all analyses.

In another set of models, we tested the effect of period, proportion of OSR and semi-natural grasslands on species-level metrics: normalized degree, species-level specialization, within and between-module connectivity, and nested rank. We fitted one model per species-level metric per country where all species of pollinators were included. Fixed factors were the same as those included in the previous set of models. 
296 We further included the abundance of each pollinator species within a site as an

297 additional fixed factor as well as its interaction with period. GLMMs were fitted with a

298 Poisson error distribution. Site was included as a random effect in all cases. All analyses

299 were performed in the glmmADMB package (Skaug et al. 2012) using R version 3.0.2

300 (R Development Core Team 2011).

301

302

303

304

305

306

307

308

309

310

311

312

313

314

315

316

317

318

319

\section{Pollinator attraction simulation}

To evaluate whether an increase in OSR cover could have an impact on network structure we simulated pollinator attraction using sites in low OSR landscapes during OSR flowering. These sites represented our spatial control, as they were assumed to harbour communities of pollinators minimally influenced by the adjacent OSR. For each network we simulated the cumulative loss of shared pollinator individuals (i.e., those belonging to species that were found within grasslands as well as within the OSR fields), and calculated network structure metrics for the resulting plant-pollinator networks including all pollinators: those shared by grasslands and crops as well as those that were never found in the crop. Each individual was given a probability of disappearing from the network based on Equation 1. Negative values of attraction probability, At (Fig. S3 in 13 out of 72 species, 8 out of 28 and 10 out of 58 species of pollinators within Germany, Sweden and the UK), representing cases in which the species was more abundant in landscapes with high covers of OSR, were given a small probability of removal $(0.001)$, while species that were never found within the crop were given a probability of 0 . We removed one pollinator individual at each time step with no replacement and continued to remove individuals until no pollinator individuals belonging to a species with an attraction probability $>0$ remained in the grassland. We ran 1,000 iterations and calculated average values for each network metric for each level of pollinator loss ( 1 to $\mathrm{N}$, where $\mathrm{N}$ is the number of shared individuals between crop and 
grassland). We then used segmented regression to identify for each site the threshold values at which each of the response variables shifted in response to the loss of pollinator individuals with package segmented in $\mathrm{R}$ version 3.0.2 (R Development Core Team 2011) with the number of segments being site-dependent. Our simulations assume there is no rewiring of interactions, meaning that when an individual pollinator is eliminated from the network its role is not occupied by another pollinator (KaiserBunbury et al. 2010). The aim of this simulation was to estimate at what point network metrics start to change in response to pollinator loss, and to compare this threshold of pollinator loss to that currently observed in our study sites. Although most network metrics are sensitive to network size (Fründ et al. 2015), the aim of this simulation exercise is to compare metrics across sites, as is done for the analyses of the robustness of networks to species loss (Memmott et al. 2004), and previous research shows that despite an overall change in network metrics, the relative order of sites is maintained for most metrics despite decreasing connectance (Bartomeus 2013). However, to control for the effect of changes in network size after species removal we ran an additional simulation where we calculated null-model corrected network metrics for 1,000 iterations following the same procedure as stated above: 1,000 null models were calculated using the vaznull algorithm. In addition, to test whether the identity of pollinator species being attracted towards the crop affected our results, in this simulation pollinator individuals were removed randomly, i.e. all species (those sampled within the crop as well as those that were never found there) had an equal

342 probability of being removed.

\section{Results}


345 We collected data from 177 networks, with >5,900 interaction events and including 223

346 pollinator species and 199 plant species (see Table S1 for values per site). The majority

347 of sampled pollinators were bumblebees (45.4\%), followed by hoverflies $(28.1 \%)$,

348 solitary bees $(15.8 \%)$ and honeybees $(10.6 \%)$. There was substantial variation in the

349 composition of the pollinator communities across countries (see Table S3). Flowering

350 plant species richness also varied between countries and periods. In general there were

351 more flowering plant species in the networks sampled after OSR flowering than during

352 flowering (Table S4A).

We found that our survey was able to capture between 61 and $99 \%$ of the

pollinator species richness in our study areas as well as 41 to $52 \%$ of the plant-pollinator

link richness (Table S5, Fig. S1), showing values similar to those found in other studies

(Chacoff et al. 2012 who used Chao2 estimates).

We found changes in species composition across years and periods for all between grasslands located in areas of high and low OSR cover were only apparent in the case of the UK (Table S6, Fig. S2). Most variation was explained by temporal changes. Hence, the pollinator communities across sites were comparable. \pm 5.3 and $19.9 \pm 6.5$ species of pollinators per site within Germany, Sweden and the UK respectively. These species represented an average of 55\% of pollinator species shared with the adjacent semi-natural grasslands (Table S4B, Fig. S3). The group of shared pollinators between the crop and the semi-natural grassland resembled closely that of

367 the pollinator community within the surveyed grasslands for each country. In Germany, 368 the pollinator community and the shared species community were both roughly evenly 
distributed across bumblebees, hoverflies and solitary bees (Table S3). In Sweden and the UK, the community of shared pollinator were dominated by hoverflies and bumblebees, respectively (Table S3). In landscapes with high OSR during flowering $8.1 \%, 26.6 \%$ and $35.3 \%$ (based on Equation 2) of pollinator individuals of species shared between the crop and the grasslands were being attracted towards the crop from grasslands in Germany, Sweden and the UK, respectively.

\section{Plant-pollinator networks}

There was a general lack of interactive effects between OSR cover and period on the network structure (Table 1, Fig. 2) and large differences between countries in how networks in each country respond to OSR flowering. In particular, link density increased after flowering in two of the three countries surveyed (with the exception of Sweden, Fig. 2 a-c) and showed a positive response to flower cover in Sweden, while the opposite was true for interaction evenness across all three countries (Figs. 2 d-f). We found the expected period:OSR cover interaction in the case of Sweden, where complementary specialization increased during the flowering pulse in landscapes with high OSR cover to decrease after. Nestedness decreased across both periods but particularly so during OSR flowering (Table 1, Fig. 3b, c). In the UK, complementary specialization $\left(\mathrm{H}^{\prime}{ }_{2}\right)$ decreased after flowering across all sites (Table 1). Modularity in Germany also responded to an interactive effect between period and the proportion of OSR in the landscape, increasing particularly during flowering in areas with greater OSR cover. Modularity showed no changes in Sweden and decreased in the UK after flowering but only in one of the years surveyed (2013). Finally, nestedness increased after flowering in Germany and the UK (Table 1). 
Patefield null models, showed some slight differences although in general showed the

same lack of interactive effects between period and the proportion of OSR in the landscape, contrary to our expectations (Tables S7-S8).

At the species level, changes in species roles within plant-pollinator networks were solely driven by changes in species abundances and period across all sampled landscapes and countries (Table 2). In general we found low values for both between and within-module connectivity with only Bombus lapidarius acting as a network hub (with c $>0.63$ and z>2.5, (Olesen et al. 2007), in a network in the UK, Fig. S4 a-c).

Nested rank, showed low values for more abundant species (i.e. generalist species) metrics we found no significant interaction between period and OSR cover for any of the metrics evaluated.

\section{Pollinator attraction simulation}

406

407

The removal of pollinator individuals from grasslands belonging to species found both at the OSR fields and grasslands (i.e., shared species) according to their probability of being attracted towards the crop (Fig. S3) led to changes in some of the network structure metrics (Fig. 4). In every case our segmented regression analyses identified threshold values at which network metrics shifted in response to individual pollinator loss, all of which well-exceeded current pollinator loss levels (Fig. 4). Yet pollinator removal did not affect all metrics equally, nor did metrics respond in the same direction across sites. Instead, changes in network structure appear highly context-dependent and a function of the identity of the initial pollinator community. In particular, link density tended to decrease across all countries (Fig. 4), while evenness remained rather stable 
and showed increases and decreases in all three countries only when large proportions of shared pollinator individuals moved to the crop (Fig. 4). Complementary specialization showed differing responses for the different countries and sites, being the metric that showed largest variability across sites. Modularity increased slightly in all three countries but particularly in the UK. However, in line with other metrics it showed large variation across sites (Fig. 4). Nestedness tended to decrease in all countries as shared pollinator individuals were extracted from the grassland network being one of the variables that most consistently responded negatively to pollinator loss (Fig. 4). A comparison with a random-removal simulation with null-model corrected metrics shows no major differences (other than site-specific differences) given that the pollinator individuals that are attracted to OSR are also the most common, abundant species. Thus, given their larger numbers they also have the greatest chances of being removed, even under a random removal scenario (Fig. S5). However, we do observe differences in the rate of change with thresholds for most metrics occurring at much lower levels of pollinator loss for random deletions.

\section{Discussion}

Our analysis across three countries of plant-pollinator interaction networks in semi-natural grasslands, during and after the flowering of OSR, showed that network structures are robust to such spatial and temporal resource fluctuations even though the crop is attracting pollinator individuals. Our results suggest that plant-pollinator networks are modified primarily by temporal changes in pollinator and plant phenology.

437 Furthermore, our pollinator-removal simulations suggest that networks are relatively resistant. Changes in some metrics were only apparent after $\sim 50 \%$ of pollinator individuals had disappeared, which far exceeded the loss of pollinators currently observed in grasslands in the countries surveyed ( $8-35 \%)$. 
The community of shared pollinator species found in the crop and the grasslands matches that of the whole pollinator community in each country. These results are expected for such a generalist plant as OSR, which attracts large numbers of opportunistic species rather than a specialized subset of species, yet the identity and impact on the pollinator community is different for each country. This is consistent with our expectations, whereby mass-flowering crops primarily attract generalist species (Kleijn et al. 2015) which reduce their relative abundance within adjacent semi-natural grasslands, but in contrast to what is observed in relation to habitat loss (Fortuna and Bascompte 2006), rare species do not seem to be directly attracted towards these crops. individuals in grasslands, landscapes with high OSR still retain a high proportion of the shared pollinators (ranging from 65\% to $92 \%$ of individuals of shared species), while major changes in pollinator communities are associated to temporal effects related to pollinator phenologies across all landscapes. The number of flowering plant species detected greatly increases in the period after flowering, suggesting that most coflowering plant species in the three countries have phenologies that do not overlap with that of OSR. Thus, it is temporal shifts such as those found for flowering plants that have an effect on network metrics.

Plant-pollinator networks

Link density increases in two of the countries, while interaction evenness decreases, in the period after crop flowering across all landscapes. This suggests that both pollinator and plant abundances increase, but that it is particular species of 
generalist pollinators that increase their abundance. This increase in generalist species after OSR flowering is also reflected in the increase in nestedness found in this period.

It is therefore not surprising that given the low levels of pollinator individual losses within our surveyed sites, network metrics do not respond to OSR flowering. Further, our simulation which sequentially removed pollinator individuals, suggests that while some metrics are robust to the loss of these relatively common species (e.g. interaction evenness), other metrics only remain relatively stable until pollinator individual loss exceeds that currently faced by our surveyed grasslands (e.g. link density or complementary specialization). However, in the case in which individuals were removed at random we find that network metrics start to change at values of individual pollinator loss that are lower than those currently found within our sites. This suggests that the relative resistance of our observed networks to pollinator loss is due to the type of pollinators being attracted to OSR: abundant and common species.

The changes observed represent a mirror image of the temporal effects observed: both link density and nestedness decrease in response to the loss of these shared generalist species. In addition we find that the progressive loss of shared pollinators could lead to further changes if OSR cover in the landscape were to increase. Of note is the effect that the loss of pollinators has on complementary specialization $\left(\mathrm{H}_{2}^{\prime}\right)$ and modularity, which although context-dependent, tend to increase with pollinator loss. This increase in complementary specialization suggests that the interactions become more exclusive and species more dependent on their partners, which raises the risk of secondary extinctions and the vulnerability of networks to further change (Blüthgen 2010, Weiner et al. 2013), although it could also increase the efficiency of pollination (Waser and Ollerton 2006). Correlated with the increase in complementary specialization is the observed decrease in nestedness which could further reduce 
network stability (Bastolla et al. 2009, Thébault and Fontaine 2010, although see, James et al. 2012), as well as the increase in modularity detected as more generalist connector species are lost and disconnected from modules (Thébault and Fontaine 2010, Spiesman and Inouye 2013). Such an increase in modularity is a consequence of disturbance also observed in other plant-pollinator networks (Spiesman and Inouye 2013, although see, Albrecht et al. 2014) and it can affect species persistence. It is worth noting, however, that we have not included rewiring within our simulations (Kaiser-Bunbury et al. 2010) - i.e. when certain pollinators are lost their function may be taken over by others - which could have attenuated some of the observed effects. However, this is probably not a limiting factor in our analyses because the species that are lost to the crop are generalist species, whose roles might not be easily filled by the remaining pollinators. Moreover, it is important to highlight that our study is restricted to diverse arable landscapes that still retain semi-natural habitat cover (2-32\%), such as forests or other grasslands which can provide nesting sites, refuges, and feeding grounds that could potentially dilute the effects of OSR on plant-pollinator networks. Finally, OSR may have long term positive effects for some species ((e.g. those where attraction probability was negative due to larger abundances within areas surrounded by high OSR covers, see also (Jauker et al. 2012)) increasing their populations at the landscape level and minimizing the impacts of a temporal attraction. Most of these results based on raw network metric values hold when comparing them to null models that control for network size and link density. However, we also note that some of these results, albeit real and measurable, are driven by the loss of species as reflected by the contrasting results of the null-corrected plantpollinator networks. This finding could be explained by the fact that the magnitude of pollinator loss suffered by semi-natural grasslands adjacent to OSR fields is dwarfed by the changes in both pollinator and plant communities due to phenology. However, we 
514 find the landscapes in different countries vary in their resistance to the expansion of

515 OSR, particularly if their pollinator community is composed of central place foragers

516 (those that depend on nests, e.g. bumblebees in the UK) as opposed to those dominated

517 by free-moving species whose life cycle depends less on floral resources (e.g. hoverflies

518 in Sweden). The resistance of networks to flowering pulses shows that the mismatching

519 phenology between OSR (which flowers in early spring) and wild plants makes the

520 abundance of OSR flowers complement rather than shift pollinator diets, boosting

521 pollinator communities with the extra resources. Overall, our study represents a step

522 towards understanding the effect of entomophilous crops on mutualistic plant-pollinator

523 networks. Nevertheless, we do not know which effect flowering crops have on

524 pollinator function (Ballantyne et al. 2015) or pollinator-dependent wild flower species

525 reproduction. Future studies should evaluate the effect of OSR and other mass-

526 flowering crops on seed set in wild plants with different flowering phenologies (e.g.

527 flowering synchronously with the crop vs. those flowering before or after the crop, cf.

528 (Kovács-Hostyánszki et al. 2013)).

\section{Acknowledgements}

530 This project was funded by the EU FP7 STEP project "Status and Trends of European

531 Pollinators" (244 090, http://www.STEP-project.net) and Biodiversa-FACCE project

532 "Enhancing biodiversity-based ecosystem services to crops through optimized densities

533 of green infrastructure in agricultural landscapes" (PCIN-2014-048,

534 http://www.cec.lu.se/research/ecodeal). AM, MV, JG-V and IB acknowledge funding

535 from the Spanish Severo Ochoa Program (SEV-2012-0262). AM acknowledges funding

536 from the Juan de la Cierva program. JBW, VJW and SGP acknowledge the support of

537 Insect Pollinators Initiative research funded jointly by a grant from BBSRC, Defra,

538 NERC, the Scottish Government and the Wellcome Trust, under the Living with 
539 Environmental Change Partnership. HGS acknowledges the support of the Formas

540 project SAPES for landscape analyses. AH received funding from DFG-Collaborative

541 Research Center 1047, Insect Timing. We thank two anonymous reviewers for

542 comments on a previous version of this manuscript. 
Table 1. Confidence intervals for estimates of variables included in the averaged models (for all models with $\Delta$ AICc values $<6$ ) for the spatial and temporal variables affecting the network level metrics in the three countries (Germany, Sweden and the UK). Fixed factors included were Period (during or after), OSR = oilseed rape proportion within $1 \mathrm{~km}, \mathrm{SNH}=$ semi-natural habitat within $1 \mathrm{~km}$, Year $(2011$ or 2012 , or, 2012 or 2013 for the UK) and Flower cover. In all cases 'during' was used as the reference category for the variable period. Bold numbers indicate cases where confidence intervals do not overlap with 0 . Missing values represent variables that were not included in final selected models.

\begin{tabular}{|c|c|c|c|}
\hline & Germany & Sweden & UK \\
\hline A) Link density & Lower CI, Upper CI & Lower CI, Upper CI & Lower CI, Upper CI \\
\hline Period & $-1.22,-0.48$ & $0.02,0.84$ & $-1.75,-0.49$ \\
\hline Proportion OSR & $-0.33,0.08$ & $-0.42,0.10$ & $0.13,0.73$ \\
\hline Proportion SNH & $-0.35,0.19$ & $-0.31,0.21$ & $-0.33,0.26$ \\
\hline Year & $-0.47,0.26$ & $-0.44,0.45$ & $-0.41,0.80$ \\
\hline Flower cover & $-0.22,0.25$ & $0.06,0.59$ & $-0.31,0.23$ \\
\hline Period : Proportion OSR & $-0.45,0.28$ & $-0.66,0.13$ & $-1.43,-0.06$ \\
\hline Period : Proportion SNH & $-0.07,0.65$ & $-0.66,0.11$ & $0.04,1.17$ \\
\hline Period : Flower cover & $-0.13,0.69$ & $-0.47,0.54$ & - \\
\hline Year : Proportion OSR & $-0.32,0.40$ & $-0.44,0.55$ & - \\
\hline Year : Proportion SNH & - & - & - \\
\hline Year: Flower cover & - & $-0.44,0.42$ & - \\
\hline \multicolumn{4}{|l|}{ B) Interaction evenness } \\
\hline Period & $0.02,0.61$ & $0.04,0.12$ & $0.02,0.21$ \\
\hline Proportion OSR & $-0.03,0.01$ & $-0.03,0.02$ & $-0.07,0.04$ \\
\hline Proportion SNH & $-0.04,0.01$ & $-0.02,0.04$ & $-0.08,0.02$ \\
\hline Year & $-0.07,0.01$ & $-0.08,0.00$ & $-0.10,0.09$ \\
\hline Flower cover & $-0.03,0.01$ & $-0.02,0.03$ & - \\
\hline Period : Proportion OSR & $-0.04,0.02$ & $-0.07,0.00$ & $-0.09,0.10$ \\
\hline Period : Proportion SNH & $-0.03,0.04$ & $-0.07,0.01$ & $-0.09,0.10$ \\
\hline & $-0.03,0.06$ & & \\
\hline Period: Flower cover & & $-0.04,0.04$ & - \\
\hline Year : Proportion OSR & $-0.05,0.02$ & $-0.04,0.04$ & - \\
\hline Year : Proportion SNH & $-0.05,0.01$ & $-0.02,0.06$ & - \\
\hline Year: Flower cover & $-0.04,0.04$ & $-0.04,0.04$ & - \\
\hline C) Complementary special & & & \\
\hline
\end{tabular}


Period

Proportion OSR

Proportion SNH

Year

Flower cover

Period : Proportion OSR

Period : Proportion SNH

Period: Flower cover

Year : Proportion OSR

Year: Proportion SNH

Year: Flower cover

\section{D) Modularity}

\section{Period}

Proportion OSR

Proportion SNH

Year

Flower cover

Period : Proportion OSR

Period : Proportion SNH

Period: Flower cover

Year : Proportion OSR

Year : Proportion SNH

Year: Flower cover

E) Nestedness

\section{Period}

Proportion OSR

Proportion SNH

Year

Flower cover

Period : Proportion OSR

Period : Proportion SNH

Period: Flower cover

Year : Proportion OSR

Year : Proportion SNH

\begin{tabular}{|c|c|c|}
\hline $\begin{array}{c}-0.06,0.17 \\
-0.04,0.07 \\
-0.03,0.09 \\
-0.01,0.21 \\
-0.05,0.08 \\
- \\
-0.12,0.10 \\
-0.04,0.24 \\
-0.10,0.11 \\
-0.07,0.14 \\
-0.16,0.07 \\
\end{array}$ & $\begin{array}{c}-0.16,0.11 \\
-0.12,0.08 \\
-0.12,0.04 \\
-0.14,0.14 \\
-0.11,0.04 \\
\mathbf{0 . 0 2 , 0 . 2 6} \\
-0.04,0.26 \\
-0.11,0.19 \\
- \\
-0.21,0.09 \\
- \\
\end{array}$ & $\begin{array}{c}\mathbf{0 . 3 4}, \mathbf{0 . 6 1} \\
-71.21,85.09 \\
-36.32,36.99 \\
-0.24,0.01 \\
-112.22,126.84 \\
-0.05,0.25 \\
-0.25,0.00 \\
-0.52,0.02 \\
-0.15,0.06 \\
-0.12,0.10 \\
-0.23,0.14 \\
\end{array}$ \\
\hline $\begin{array}{c}-0.12,0.02 \\
-0.04,0.06 \\
-0.05,0.03 \\
-0.06,0.07 \\
-0.03,0.04 \\
\mathbf{0 . 0 0 , 0 . 1 3} \\
-0.09,0.06 \\
-0.05,0.14 \\
-0.08,0.06 \\
- \\
- \\
\end{array}$ & $\begin{array}{c}-0.09,0.07 \\
-0.03,0.06 \\
-0.01,0.09 \\
-0.02,0.15 \\
-0.07,0.03 \\
- \\
-0.12,0.07 \\
-0.15,0.03 \\
-0.07,0.11 \\
-0.12,0.05 \\
-0.11,0.06 \\
\end{array}$ & $\begin{array}{c}\mathbf{0 . 0 2}, \mathbf{0 . 2 0} \\
-38.82,43.66 \\
-48.92,55.22 \\
\mathbf{- 0 . 1 8 ,},-\mathbf{0 . 0 1} \\
-82.72,90.42 \\
-0.14,0.02 \\
-0.02,0.14 \\
-0.37,0.00 \\
-0.10,0.06 \\
-0.10,0.06 \\
-0.16,0.12 \\
\end{array}$ \\
\hline$-0.81,-0.24$ & $-1.10,-0.51$ & $-1.85 \mathrm{e}+05,1.77 \mathrm{e}+05$ \\
\hline $\begin{array}{c}-0.26,0.12 \\
-0.11,0.35 \\
-\mathbf{0 . 5 7}, \mathbf{- 0 . 1 1} \\
-0.04,0.33 \\
-0.49,0.10 \\
\mathbf{0 . 1 0 , 0 . 6 7} \\
\mathbf{0 . 1 0 , 0 . 8 1}\end{array}$ & $\begin{array}{c}\mathbf{- 1 . 2 9 ,}, \mathbf{- 0 . 5 0} \\
- \\
-0.15,0.54 \\
\mathbf{0 . 0 2}, \mathbf{0 . 5 1} \\
\mathbf{0 . 0 6}, \mathbf{0 . 7 3} \\
- \\
\mathbf{- 1 . 0 2 ,}-\mathbf{0 . 3 1}\end{array}$ & $\begin{array}{c}-5.43,3.37 \\
-2.67 \mathrm{e}+02,4.03 \mathrm{e}+02 \\
\mathbf{2 . 5 9 e - 0 1 , ~ 1 . 0 5 e + 0 0} \\
-1.35 \mathrm{e}+03,7.65 \mathrm{e}+02\end{array}$ \\
\hline $\begin{array}{l}-0.06,0.41 \\
\mathbf{- 0 . 6 8 ,}, \mathbf{- 0 . 1 7}\end{array}$ & $\begin{array}{l}- \\
-\end{array}$ & $\begin{array}{l}-2.59 \mathrm{e}-01,6.97 \mathrm{e}-01 \\
-3.53 \mathrm{e}-01,1.30 \mathrm{e}-01\end{array}$ \\
\hline
\end{tabular}


Table 2. Confidence intervals for estimates of variables included in the averaged models (for all models with $\Delta$ AICc values $<6$ ) for the spatial and temporal variables affecting the species level network metrics in the three countries (Germany, Sweden and the UK). In all cases 'during' was used as the reference category for the variable period. Bold numbers indicate cases where confidence intervals do not overlap with 0.

Missing values represent variables that were not included in final selected models.

\begin{tabular}{|c|c|c|c|}
\hline & Germany & Sweden & UK \\
\hline A) Normalised degree & Lower CI, Upper CI & Lower CI, Upper CI & Lower CI, Upper CI \\
\hline Period & $0.44,1.40$ & $-0.02,0.75$ & $-0.06,1.78$ \\
\hline Abundance & $0.09,0.33$ & $-0.02,0.22$ & $0.08,0.40$ \\
\hline Proportion OSR & $-0.28,0.2$ & $-0.22,0.21$ & $-0.29,0.31$ \\
\hline Proportion SNH & $-0.22,0.24$ & $-0.28,0.15$ & $-0.36,0.26$ \\
\hline Year & $-0.35,0.49$ & $-0.60,0.16$ & $-0.57,0.65$ \\
\hline Period : Abundance & $-1.13,1.29$ & $-0.14,1.07$ & $-2.26,4.35$ \\
\hline Period : Proportion OSR & $-0.59,0.27$ & $-0.33,0.41$ & $-0.80,0.60$ \\
\hline Period: Proportion SNH & $-0.50,0.40$ & $-0.33,0.43$ & $-0.70,0.76$ \\
\hline Year : Proportion OSR & $-0.57,0.27$ & $-0.41,0.40$ & - \\
\hline Year : Proportion SNH & $-0.41,0.45$ & $-0.42,0.35$ & $-0.73,0.49$ \\
\hline \multicolumn{4}{|c|}{ B) Species-level specialization (d') } \\
\hline Period & $-2.58,-0.15$ & $-0.88,0.48$ & $-0.21,0.11$ \\
\hline Abundance & $-1.40,-0.13$ & $-2.06,0.23$ & $-0.06,0.00$ \\
\hline Proportion OSR & $-0.39,0.14$ & $-0.39,0.30$ & $-0.02,0.06$ \\
\hline Proportion SNH & $-0.34,0.17$ & $-0.34,0.33$ & $-0.02,0.05$ \\
\hline Year & $-0.41,0.55$ & $-0.68,0.62$ & $-0.08,0.04$ \\
\hline Period : Abundance & $-3.88,4.67$ & $-1.75,2.91$ & $-0.20,0.85$ \\
\hline Period : Proportion OSR & $-0.85,0.94$ & $-1.06,0.31$ & $-0.13,0.05$ \\
\hline
\end{tabular}




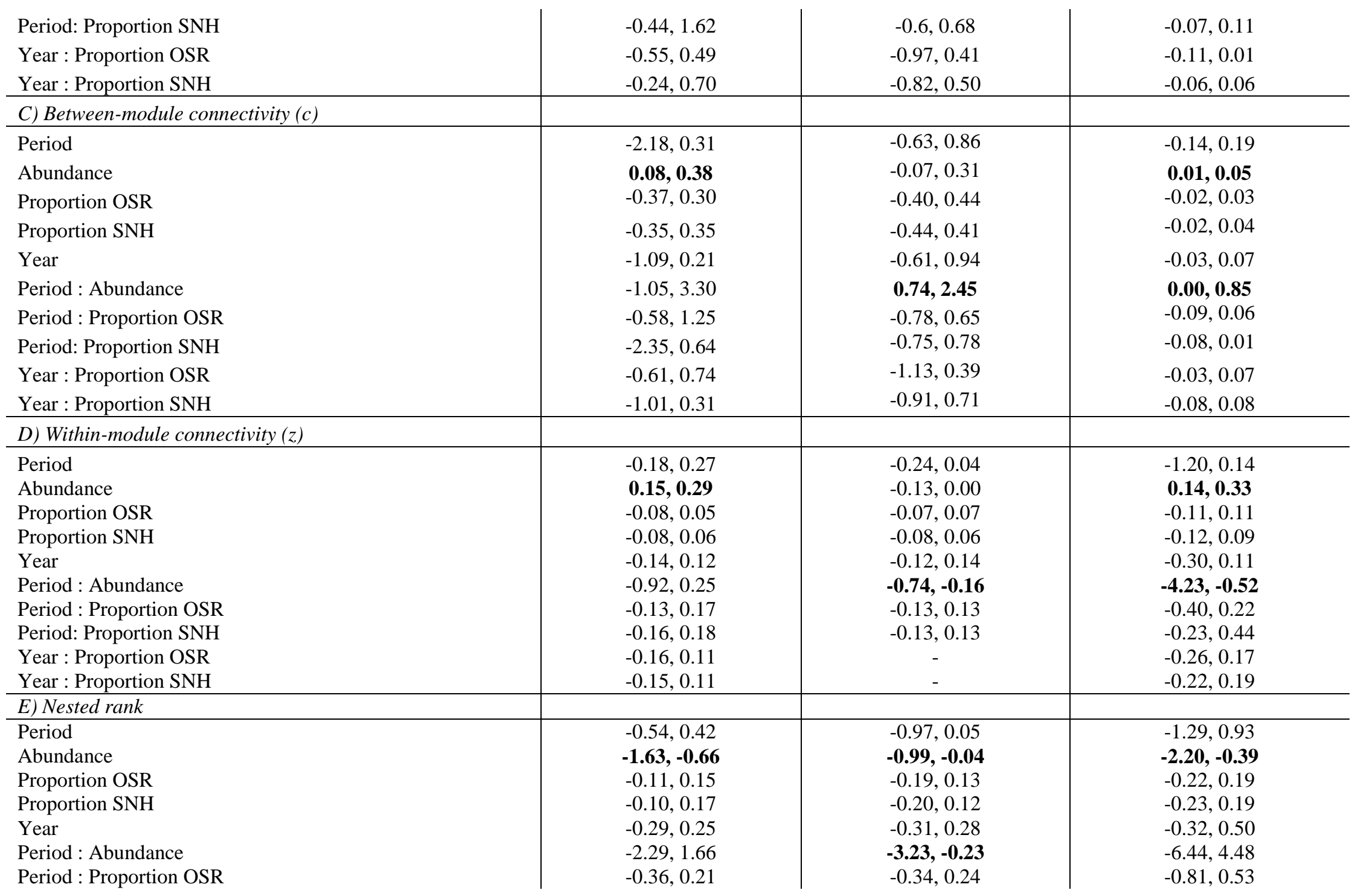


Period: Proportion SNH

Year : Proportion OSR

$-0.26,0.40$

$-0.23,0.35$

$-0.30,0.31$ 
556 Figure 1. a) Location of study sites across the three countries sampled. b) Schematic

557 representation of the study design showing the number of sites sampled at each

558 landscape type-period combination. c) Expectation in pollinator abundances during and

559 after OSR flowering in the crop and semi-natural grasslands. During flowering OSR is

560 expected to attract common and generalist species which will see their abundances

561 decrease within semi-natural grasslands surrounded by high OSR proportions. These

562 pollinators are then expected to return to the grasslands after the crop has ceased

563 flowering, while no apparent changes are expected within grasslands surrounded by low

564 OSR proportions. The change in pollinator abundance in grasslands surrounded by high

565 OSR proportions during crop blooming is reflected in lost links in the semi-natural

566 grassland plant-pollinator network.

567 Figure 2. Boxplots showing the effect of period (during and after oilseed rape

568 flowering, OSR) on link density and interaction evenness in nearby semi-natural

569 grasslands for the three countries. Boxes around median extend from first to third

570 quartiles. Inset in top panels shows examples of real networks for each country and

571 period. Brown filled circles represent pollinator species, and grey filled circles plant

572 species.

573 Figure 3. Partial residual plot showing the interactive effect between the scaled

574 proportion of oilseed rape and period on modularity in Germany and complementary

575 specialization and nestedness in Sweden.

576

577 Figure 4. Results of simulations showing the effect of extracting individuals belonging

578 to shared pollinator species from control sites (landscapes with low or no oilseed rape 
579 cover (OSR) during oilseed rape flowering) on different network metrics for Germany

580 a)-e), Sweden f)-j) and the UK k)-o). Black dashed line indicates the mean proportion of 581 shared pollinator species that are lost in landscapes of high OSR for each country based 582 on Equation $2(8.1 \%, 26.6 \%$ and $35.3 \%$ for Germany, Sweden and the UK

583 respectively). Different coloured lines indicate segmented regression fits for different

584 sites pooled across both study years. Networks in some cases were too small to compute

585 some of the metrics and are not shown in the figure. In cases where we were unable to

586 find breakpoints using segmented regression, we present linear regressions instead. 

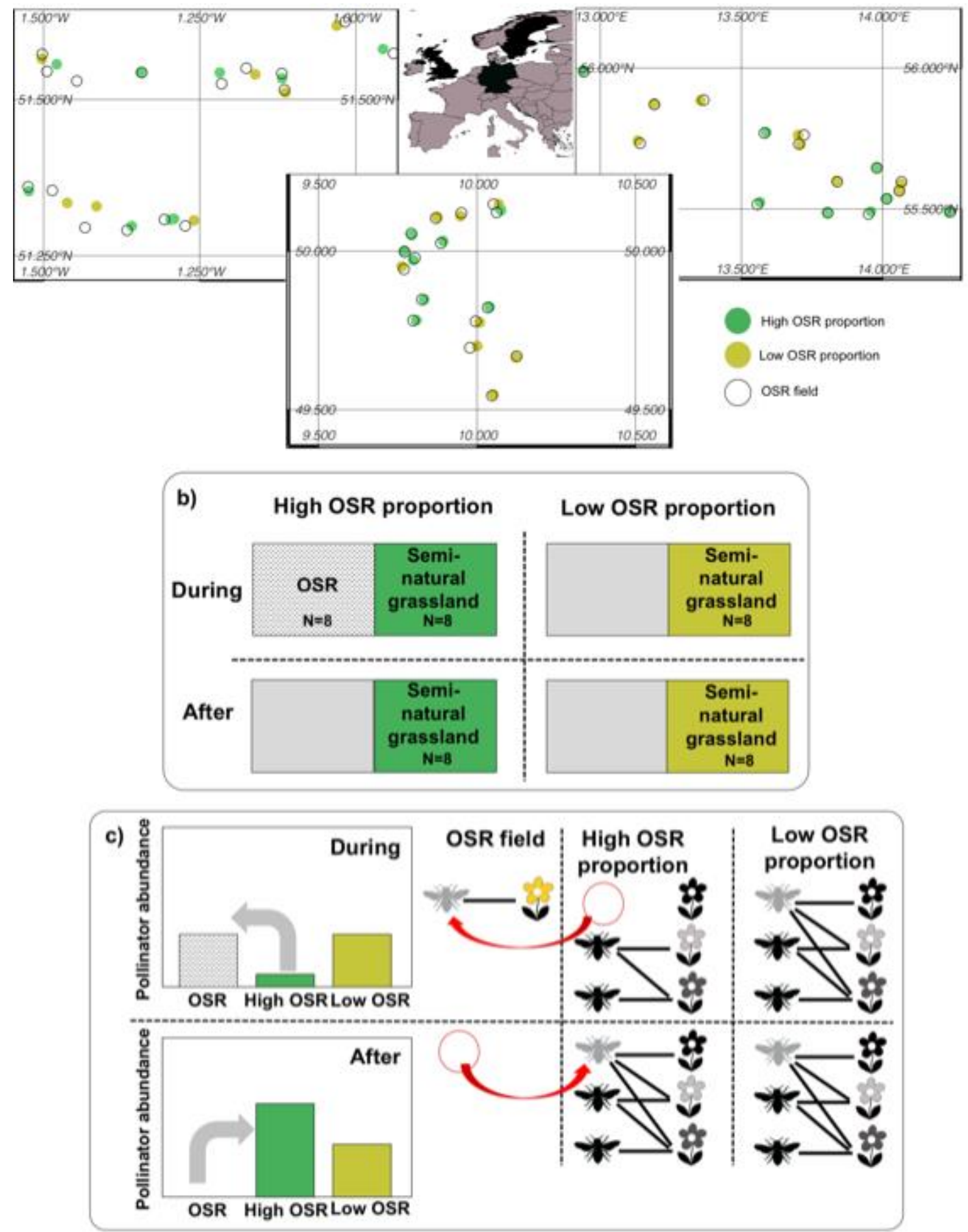

Figure 1. 


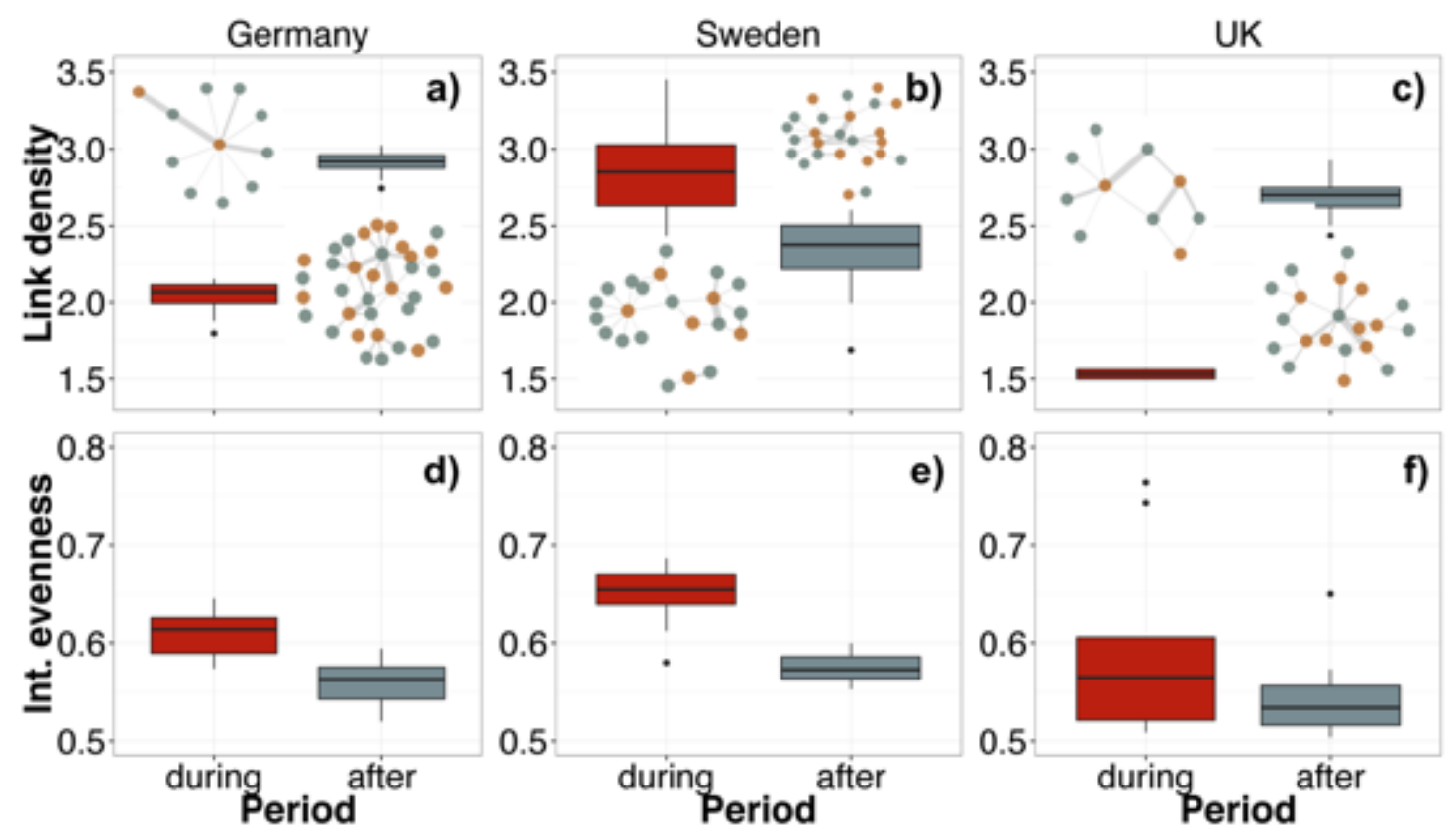

591

592

Figure 2.

593 

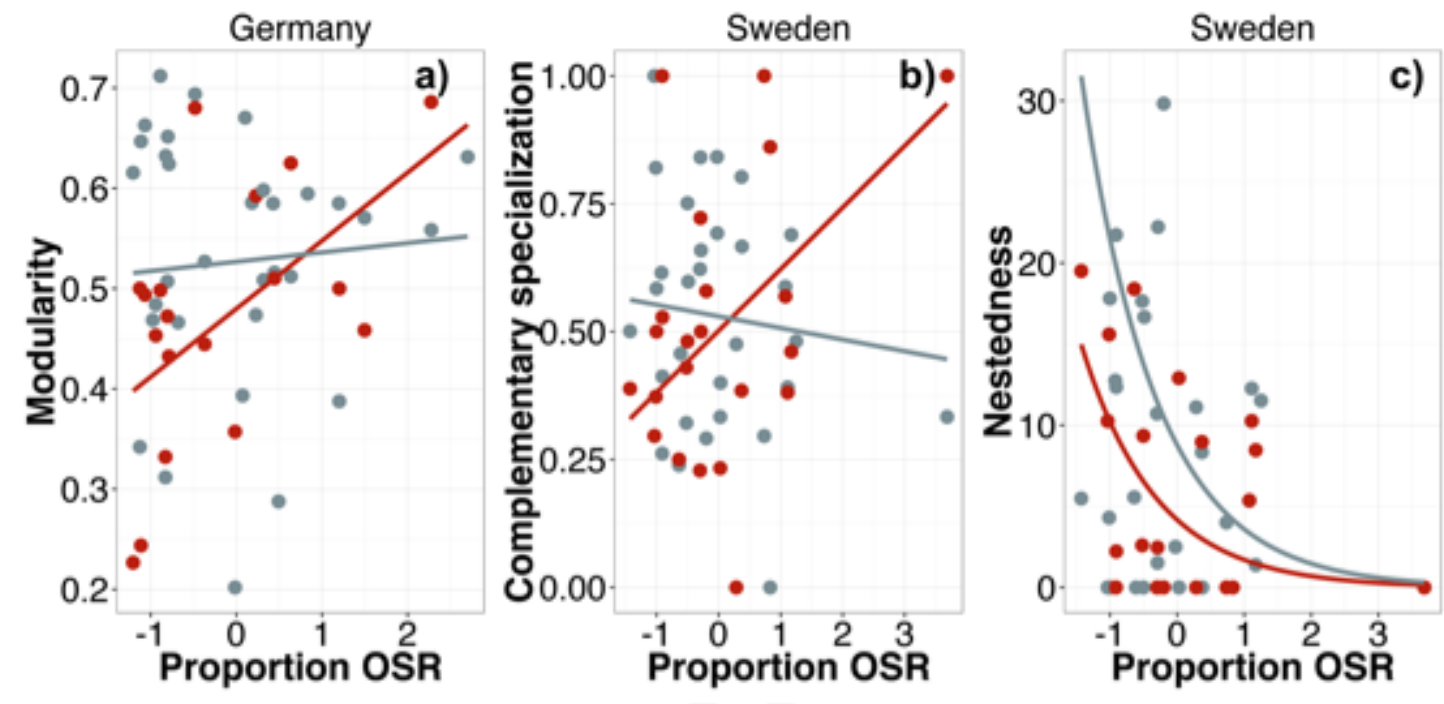

595

Figure 3. 

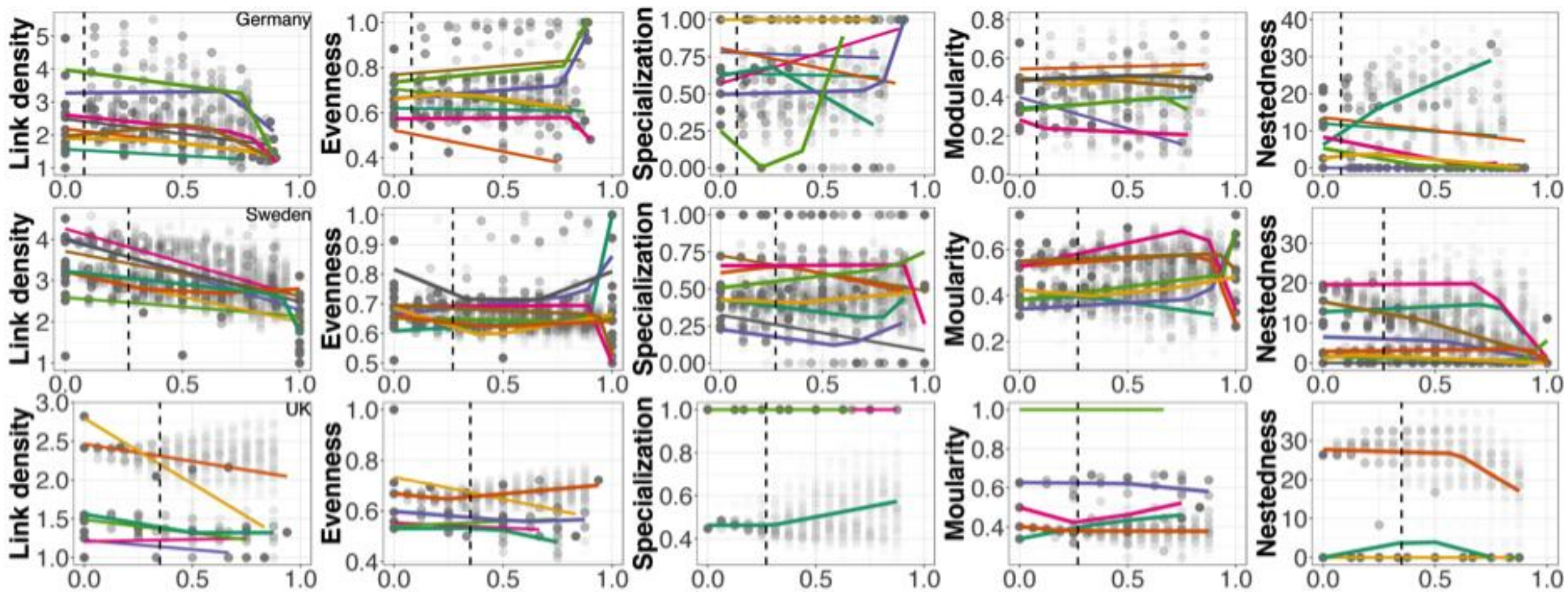

Pollinator individuals lost

Figure 4. 
601

602

603

604

605

606

607

608

609

610

611

612

613

614

615

616

617

618

619

620

621

622

623

624

Aizen, M. A. et al. 2012. Specialization and Rarity Predict Nonrandom Loss of Interactions from Mutualist Networks. - Science (80-. ). 335: 1486 LP - 1489.

Alarcón, R. et al. 2008. Year-to-year variation in the topology of a plant-pollinator interaction network. - Oikos 117: 1796-1807.

Albrecht, M. et al. 2014. Consequences of plant invasions on compartmentalization and species' roles in plant-pollinator networks. - Proc. R. Soc. London B Biol. Sci. 281: 20140773 .

Almeida-Neto, M. and Ulrich, W. 2011. A straightforward computational approach for measuring nestedness using quantitative matrices. - Env. Model Softw 26: 173178.

Ballantyne, G. et al. 2015. Constructing more informative plant - pollinator networks : visitation and pollen deposition networks in a heathland plant community. - Proc. R. Soc. B 282: 20151130.

Bartomeus, I. 2013. Understanding Linkage Rules in Plant-Pollinator Networks by Using Hierarchical Models That Incorporate Pollinator Detectability and Plant Traits. - PLoS One 8: e69200.

Bartomeus, I. et al. 2016. A common framework for identifying linkage rules across different types of interactions. - Funct. Ecol. in press.

Bartoń, K. 2013. \{MuMIn $\}$ : multi-model inference, $\{\mathrm{R}\}$ package version 1.9.13 OR CRAN http://CRAN.R-project.org/package=MuMIn.

Bascompte, J. and Jordano, P. 2007. Plant-Animal Mutualistic Networks: The Architecture of Biodiversity. - Annu Rev Ecol Evol S 38: 567-593.

Bascompte, J. et al. 2003. The nested assembly of plant-animal mutualistic networks. P. Natl. Acad. Sci. USA 100: 9383-9387. 
Bastolla, U. et al. 2009. The architecture of mutualistic networks minimizes competition and increases biodiversity. - Nature 458: 1018-1020.

Bersier, L.-F. et al. 2002. Quantitative Descriptors of Food-Web Matrices. - Ecology 83: 2394-2407.

Blüthgen, N. 2010. Why network analysis is often disconnected from community ecology: A critique and an ecologist's guide. - Basic Appl. Ecol. 11: 185-195.

Blüthgen, N. et al. 2006. Measuring specialization in species interaction networks. BMC Ecol. 6: 1-12.

Blüthgen, N. et al. 2007. Specialization, Constraints, and Conflicting Interests in Mutualistic Networks. - Curr Biol 17: 341-346.

Burnham, K. P. et al. 2011. AIC model selection and multimodel inference in behavioral ecology: some background, observations, and comparisons. - Behav. Ecol. Sociobiol. 65: 23-35.

Chacoff, N. P. et al. 2012. Evaluating sampling completeness in a desert plant-pollinator network. - J. Anim. Ecol. 81: 190-200.

Chao, A. 1984. Nonparametric estimation of the number of classes in a population. Scand. J. Stat. 11: 265-270.

Clarke, K. R. and Warwick, R. M. 2001. Change in marine communities: an approach to statistical analysis and interpretation. 2nd edition. - Primer-E.

Diekötter, T. et al. 2010. Oilseed rape crops distort plant-pollinator interactions. - J. Appl. Ecol. 47: 209-214.

Dormann, C. F. and Strauss, R. 2014. A method for detecting modules in quantitative bipartite networks. - Methods Ecol. Evol. 5: 90-98.

Dormann, C. F. et al. 2009. Indices, graphs and null models: analyzing bipartite ecological networks. - Open Ecol. J. 2: 7-24. 
FAO 2008. The State of Food and Agriculture Biofuels: Prospects, Risks and Opportunities. (FAO, Ed.).

FAO 2009. How to feed the world in 2050 (HLEF-H to $F$ the $\mathrm{W}$ in 2050, Ed.).

FAO 2014. FAOSTAT: Statistical Databases and Data-Sets.

Foley, J. A. et al. 2005. Global consequences of land use. - Science (80-. ). 309: 570574.

Fortuna, M. A. and Bascompte, J. 2006. Habitat loss and the structure of plant-animal mutualistic networks. - Ecol Lett 9: 278-283.

Fründ, J. et al. 2015. Sampling bias is a challenge for quantifying specialization and network structure: lessons from a quantitative niche model. - Oikos 125: 502-513.

Gonzalez-Varo, J. P. et al. 2013. Combined effects of global change pressures on animal-mediated pollination. - Trends Ecol. Evol. 28: 524-530.

Guimarães, P. R. et al. 2011. Evolution and coevolution in mutualistic networks. - Ecol. Lett. 14: 877-885.

Hanke, S. et al. 2014. Landscape configuration of crops and hedgerows drives local syrphid fly abundance. - J. Appl. Ecol. 51: 505-513.

Holzschuh, A. et al. 2011. Expansion of mass-flowering crops leads to transient pollinator dilution and reduced wild plant pollination. - Proc. R. Soc. London B Biol. Sci. in press.

Holzschuh, A. et al. 2013. Mass-flowering crops enhance wild bee abundance. Oecologia 172: 477-484.

Holzschuh, A. et al. 2016. Mass-flowering crops dilute pollinator abundance in agricultural landscapes across Europe. - Ecol. Lett.: n/a-n/a.

Hoyle, M. et al. 2007. Effect of pollinator abundance on self-fertilization and gene flow: Application to GM canola. - Ecol. Appl. 17: 2123-2135. 
Hsieh, T. C. et al. 2016. iNEXT: iNterpolation and EXTrapolation for species diversity. $\mathrm{R}$ package version 2.0.8. in press.

James, A. et al. 2012. Disentangling nestedness from models of ecological complexity. Nature 487: 227-230.

Jauker, F. et al. 2012. Early reproductive benefits of mass-flowering crops to the solitary bee Osmia rufa outbalance post-flowering disadvantages. - Basic Appl. Ecol. 13: 268-276.

Jordano, P. et al. 2003. Invariant properties in coevolutionary networks of plant-animal interactions. - Ecol. Lett. 6: 69-81.

Kaiser-Bunbury, C. N. and Blüthgen, N. 2015. Integrating network ecology with applied conservation : a synthesis and guide to implementation. - AoB Plants Spec. ISSUE Isl. Plant Biol. — Celebr. Carlquist's Leg. in press.

Kaiser-Bunbury, C. N. et al. 2010. The robustness of pollination networks to the loss of species and interactions: a quantitative approach incorporating pollinator behaviour. - Ecol. Lett. 13: 442-452.

Kleijn, D. et al. 2015. Delivery of crop pollination services is an insufficient argument for wild pollinator conservation. - Nat. Commun. 6: 7414.

Koh, L. P. 2007. Potential Habitat and Biodiversity Losses from Intensified Biodiesel Feedstock Production. - Conserv. Biol. 21: 1373-1375.

Kovács-Hostyánszki, A. et al. 2013. Contrasting effects of mass-flowering crops on bee pollination of hedge plants at different spatial and temporal scales. - Ecol. Appl. 23: 1938-1946.

Magurran, A. E. 2004. Measuring biological diversity. - In: Ltd., Blackwell Science, Oxford, UK, in press.

Memmott, J. et al. 2004. Tolerance of pollination networks to species extinctions. - 
Murray, T. E. et al. 2008. Cryptic species diversity in a widespread bumble bee complex revealed using mitochondrial DNA RFLPs. - Conserv. Genet. 9: 653-666.

703

704

705

706

707

708

709

710

711

712

713

714

715

716

717

718

719

720

721

722

723

724

Nielsen, A. and Totland, Ø. 2014. Structural properties of mutualistic networks withstand habitat degradation while species functional roles might change. - Oikos 123: 323-333.

Olesen, J. M. et al. 2007. The modularity of pollination networks. - P. Natl. Acad. Sci. USA 104: 19891-19896.

Olesen, J. M. et al. 2008. Temporal dynamics in a pollination network. - Ecology 89: $1573-1582$.

Patefield, W. M. 1981. An efficient method of generating random RxC tables with given row and column totals. - Appl Stat 30: 91-97.

R Development Core Team, R. 2011. R: A Language and Environment for Statistical Computing (RDC Team, Ed.). - R Found. Stat. Comput. 1: 409.

Schleuning, M. et al. 2012. Specialization of Mutualistic Interaction Networks Decreases toward Tropical Latitudes. - Curr. Biol. 22: 1925-1931.

Schleuning, M. et al. 2014. At a loss for birds: insularity increases asymmetry in seeddispersal networks. - Glob. Ecol. Biogeogr. 23: 385-394.

Skaug, H. et al. 2012. Generalized Linear Mixed Models using AD Model Builder. . - R Packag. version 0.7.2.12 in press.

Spiesman, B. J. and Inouye, B. D. 2013. Habitat loss alters the architecture of plant pollinator interaction networks. - Ecology 94: 2688-2696.

Steffan-Dewenter, I. and Kuhn, A. 2003. Honeybee foraging in differentially structured landscapes. - Proc. R. Soc. London B Biol. Sci. 270: 569-575.

Thébault, E. and Fontaine, C. 2010. Stability of Ecological Communities and the 
Tiedeken, E. J. and Stout, J. C. 2015. Insect-Flower Interaction Network Structure Is

727 Resilient to a Temporary Pulse of Floral Resources from Invasive Rhododendron ponticum. - PLoS One: e0119733.

729

730

731

732

733

734

735

736

737

738

739

Tylianakis, J. et al. 2007. Habitat modification alters the structure of tropical hostparasitoid food webs. - Nature 445: 202-205.

Waser, N. M. and Ollerton, J. 2006. Plant-pollinator interactions. From specialization to generalization. - The University of Chicago Press.

Weiner, C. N. et al. 2013. Land-use impacts on plant-pollinator networks : interaction strength and specialization predict pollinator declines. - Ecology 95: 466-474.

Westphal, C. et al. 2003a. Mass flowering crops enhance pollinator densities at a landscape scale. - Ecol. Lett. 6: 961-965.

Westphal, C. et al. 2003b. Mass flowering crops enhance pollinator densities at a landscape scale. - Ecol. Lett. 6: 961-965. 